Article

\title{
Synthesis and Analysis of Some Bis-Heterocyclic Compounds Containing Sulphur
}

\author{
Yahia Nasser Mabkhoot
}

Department of Chemistry, King Saud University P.O Box 2455, Riyadh-11451, Saudi Arabia; E-mail: yahia@ksu.edu.sa

Received: 1 April 2009; in revised form: 15 May 2009 / Accepted: 19 May 2009 /

Published: 22 May 2009

\begin{abstract}
A facile and convenient synthesis of a series of bisheterocycles 7a,b 10, 12 and 13a,b containing a thieno[2,3-b]thiophene base unit via the versatile, hitherto unreported 3[3,4-dimethyl-5-(3-nitrilopropanoyl)thieno[2,3-b]thiophen-2-yl]-3-oxopropanenitrile (4) is described.
\end{abstract}

Keywords: DMF-DMA; bis-thieno[2,3-b]thiophene; bis-aminopyrazole; bispyrazolopyrimidine

\section{Introduction}

Thiophene derivatives represent a class of important and well-studied heterocycles [1,2]. The interest in this kind of heterocycles has spread from the early dye chemistry to modern drug design, biodiagnostics, electronic and optoelectronic devices, conductivity-based sensors, and self-assembled superstructures [3-8]. Recently, some conjugated thieno-thiophenes, structurally related to several current applications have been reported [9-15].

Thienothiophene derivatives have been developed for different purposes in the pharmaceutical field and have been tested as potential antitumor, antiviral, antibiotic, and antiglaucoma drugs, or as inhibitors of platelet aggregation [16-20]. Recently Mashraqui [10] described the first application of thieno[2,3-b]thiophene in the design of a novel NLO system by incorporating this nucleus within an unsymmetrically functionalized cyclophane. On the other hand, pyrazolopyrimidine compounds have been found to be useful as pharmaceutically interesting compounds [21,22].

However, little is known in the literature about thienothiene derivatives with different features and applications, and there is no report of a generally useful synthesis of thienothiene derivatives, 
compounds which are of considerable interest as potential biological active compounds or pharmaceuticals. In light of these findings we report here the synthesis of some novel bis-heterocycles containing thieno[2,3-b]thiophene as a base unit.

\section{Results and Discussion}

The synthetic procedures adopted to obtain the target compounds are depicted in Schemes 1, 2 and 3. Treatment of 3,4-dimethylthieno[2,3-b]thiophene-2,5-dicarboxylate (1) with acetonitrile in the presence of sodium hydride in refluxing benzene afforded the novel 3-[3,4-dimethyl-5-(3nitrilopropanoyl)thieno[2,3-b]thiophen-2-yl]-3-oxopropanenitrile (4) (Scheme 1).

Scheme 1. Synthesis of bis-aminopyrazoles derivatives 7a-b.<smiles>CCOC(=O)c1sc2sc(C(=O)OCC)c(C)c2c1C</smiles>

1

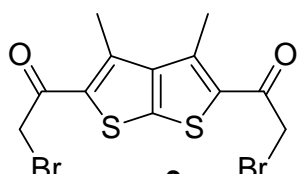

3

$\mathrm{KCN}$

$\mathrm{C}_{2} \mathrm{H}_{5} \mathrm{OH}$

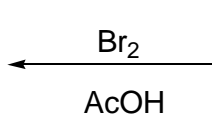

$\mathrm{AcOH}$

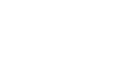

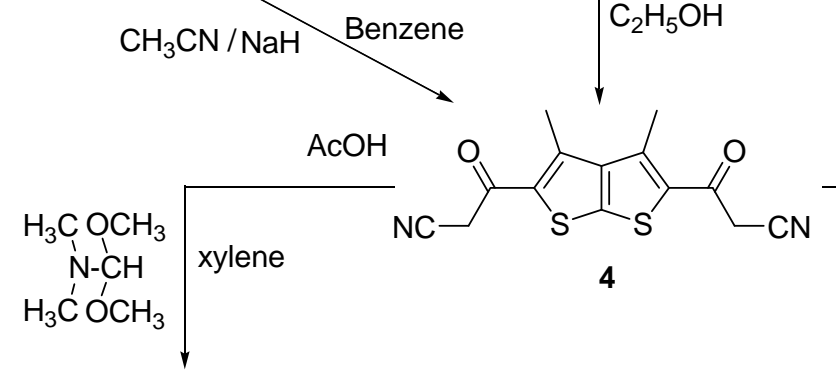
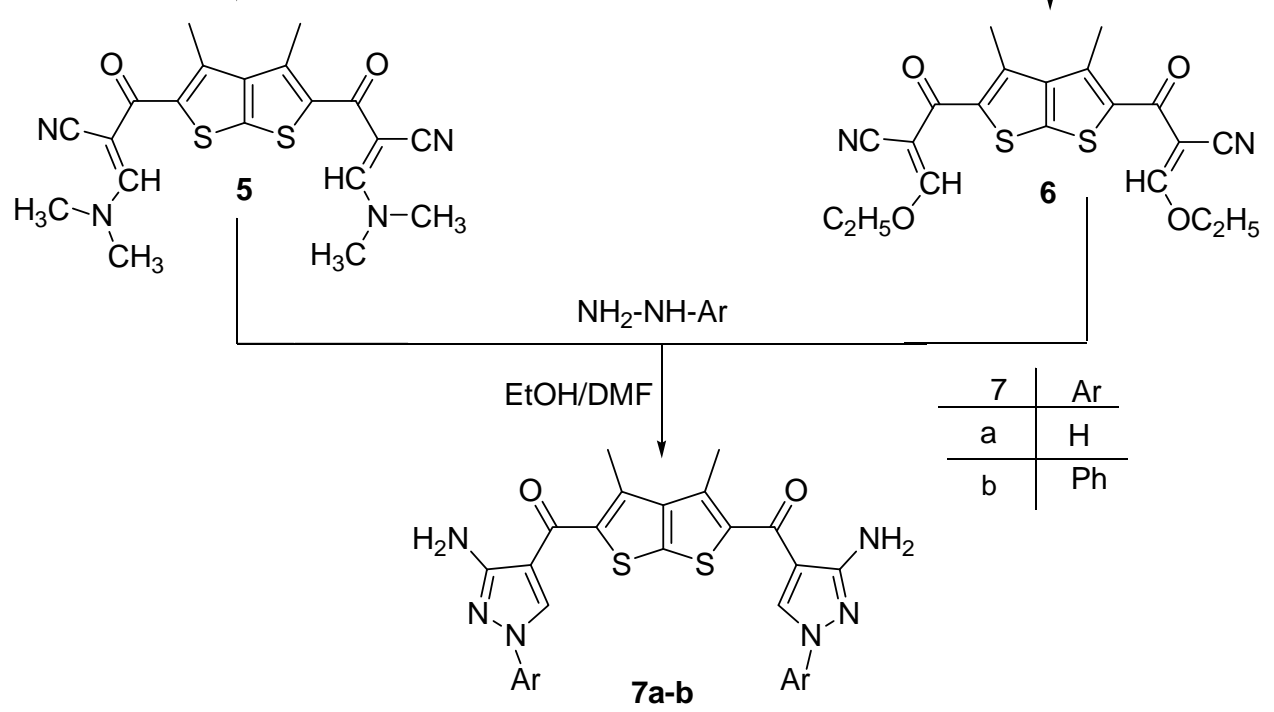

The latter could alternatively be obtained by reaction of bis-2-bromoacetylthieno[2,3-b]thiophene derivative 3 with ethanolic potassium cyanide solution. Compound 3 in turn can be obtained from 1-(5-acetyl-3,4-dimethythieno[2,3-b]thiophene-2-yl)ethanone (2) upon treatment of $\mathrm{Br}_{2}$ in $\mathrm{AcOH}$. The structure of compound 4 was established on the basis of its elemental analyses and spectral data. Its ${ }^{1} \mathrm{H}$ - NMR spectrum displayed a singlet signal at $\delta 4.03 \mathrm{ppm}$, characteristic of active methylene protons, whereas its IR spectrum revealed two absorption bands at 2,214 and $1,709 \mathrm{~cm}^{-1}$ due to nitrile and carbonyl functions, respectively. 
Treatment of compound 4 with dimethylformamide-dimethylacetal (DMF-DMA) in refluxing xylene afforded 2-(\{5-[-2-cyano-3-(dimethylamino)-2-propenoyl]-3,4-dimethylthieno[2,3-b]thiophen2-yl\} carbonyl)-3-(dimethylamino)-2-propenenitrile (5) (Scheme 1). The ${ }^{1} \mathrm{H}-\mathrm{NMR}$ spectrum of compound 5 displayed a singlet signal at $\delta 2.23$ due to methyl protons, a singlet signal at $\delta 2.47$ due to $N, N$-dimethyl protons and a singlet signal at $\delta 7.51$ due to ethylenic proton. The mass spectrum revealed a molecular ion peak at $\mathrm{m} / \mathrm{z} 412$, corresponding to $\mathrm{C}_{20} \mathrm{H}_{20} \mathrm{~N}_{4} \mathrm{O}_{2} \mathrm{~S}_{2}$. In a similar manner, when 4 was treated with excess triethylorthoformate, the corresponding bis-ethoxypropenenitrile 6 was obtained in high yield. When compounds $\mathbf{5}$ and $\mathbf{6}$ were treated with hydrazine hydrate and with phenyl hydrazine in refluxing ethanol/DMF, the novel bis(aminopyrazoles) 7a,b, were obtained, respectively (Scheme 1). The structures of the latter products were deduced from their elemental analyses and spectral data. The ${ }^{1} \mathrm{H}-\mathrm{NMR}$ spectrum of compound 7a, for example, revealed a singlet signal at $\delta 7.52$, characteristic of a pyrazole $\mathrm{CH}$ proton.

Prompted by the aforementioned results, we have also investigated the reactivity of $\mathbf{5}$ and $\mathbf{6}$ towards 5-amino-3-phenyl-1H-pyrazole (8a). Thus, reaction of $\mathbf{5}$ and $\mathbf{6}$ with this compound in refluxing ethanol/DMF, in the presence of piperidine gave 6-[5-(7-cyanopyrazolo[1,5-a]pyrimidin-6-yl)-3,4dimethylthieno[2,3-b]thiophen-2-yl]-2-phenylpyrazolo[1,5-a]pyrimidine-7-carbonitrile (10) (Scheme 2). The structure of the obtained product was assigned as $\mathbf{1 0}$ and not the other expected derivative $\mathbf{9}$ based on spectral data. The IR spectrum of the reaction product revealed, in each case, no bands due to amino or carbonyl functions. Moreover, the ${ }^{1} \mathrm{H}$ NMR spectrum of compound 10, revealed two singlet signals at $\delta 7.79$ and 8.9 due to pyrazole and pyrimidine $\mathrm{CH}$ protons, respectively. The formation of product $\mathbf{1 0}$ is assumed to take place via the addition of the exocyclic amino group in 5-amino-3phenyl-1H-pyrazole (8a) to the activated double bond in compounds 5 or $\mathbf{6}$ which then undergo intramolecular cyclization and subsequent aromatization via the loss of dimethylamine and water molecules under the reaction conditions to give $\mathbf{1 0}$ as depicted in Scheme 2.

Scheme 2. Synthesis of bis-pyrazolo[1,5-a]pyrimidine derivative $\mathbf{1 0}$.

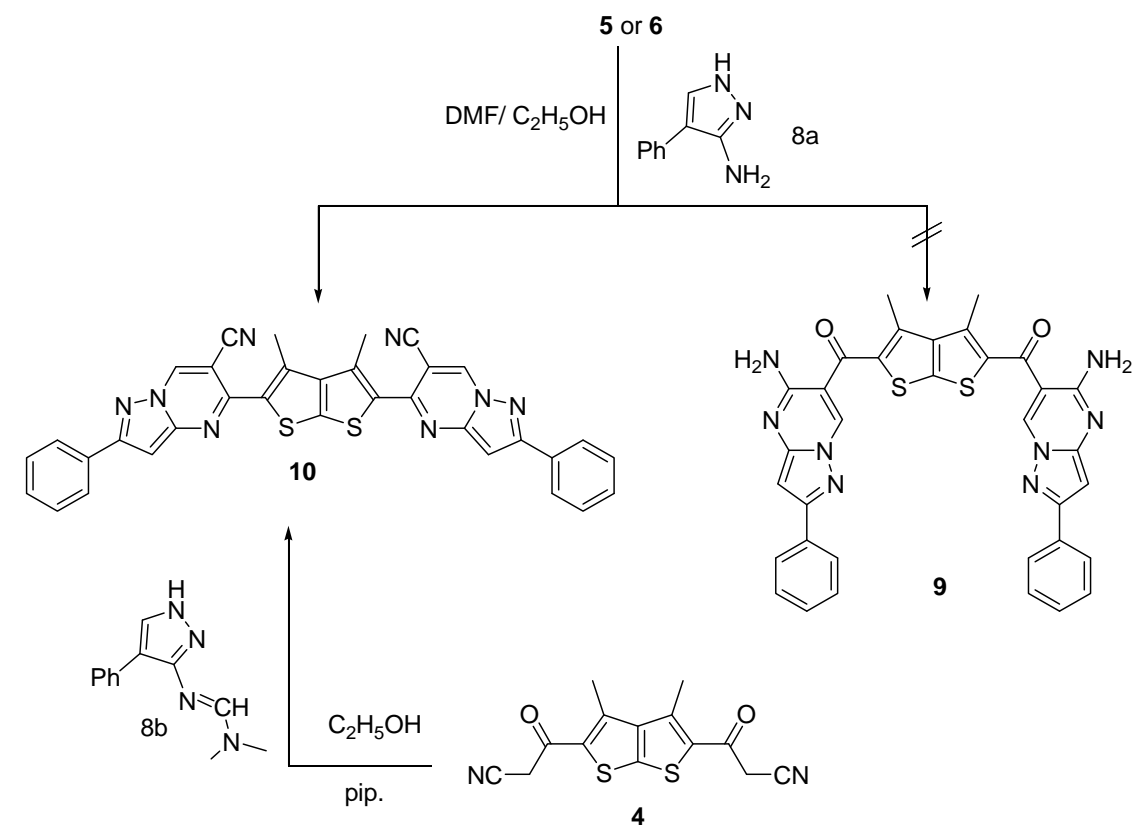


Further evidence for the proposed structure $\mathbf{1 0}$ was obtained by treatment of 5-( $N, N$ dimethylaminomethylene)imino-3-phenyl-1H-pyrazole (8b) [23] with $\mathbf{4}$ in ethanol/DMF in the presence of a catalytic amount of piperidine to afford a product indentical in all respects with that obtained from the reaction of 5 or 6 with 5-amino-3-phenyl-1H-pyrazole (Scheme 2).

Reactions of 4 with aromatic diazonium salts were also investigated. Thus, treatment of bis-3oxopropanenitrile 4 with diazotized aromatic amines (aniline and p-chloroaniline) in cold ethanol/DMF, in the presence of sodium acetate trihydrate afforded the corresponding hydrazone derivatives 11a,b (Scheme 3).

Scheme 3. Synthesis of bis-amino pyrazole derivatives 13a,b.

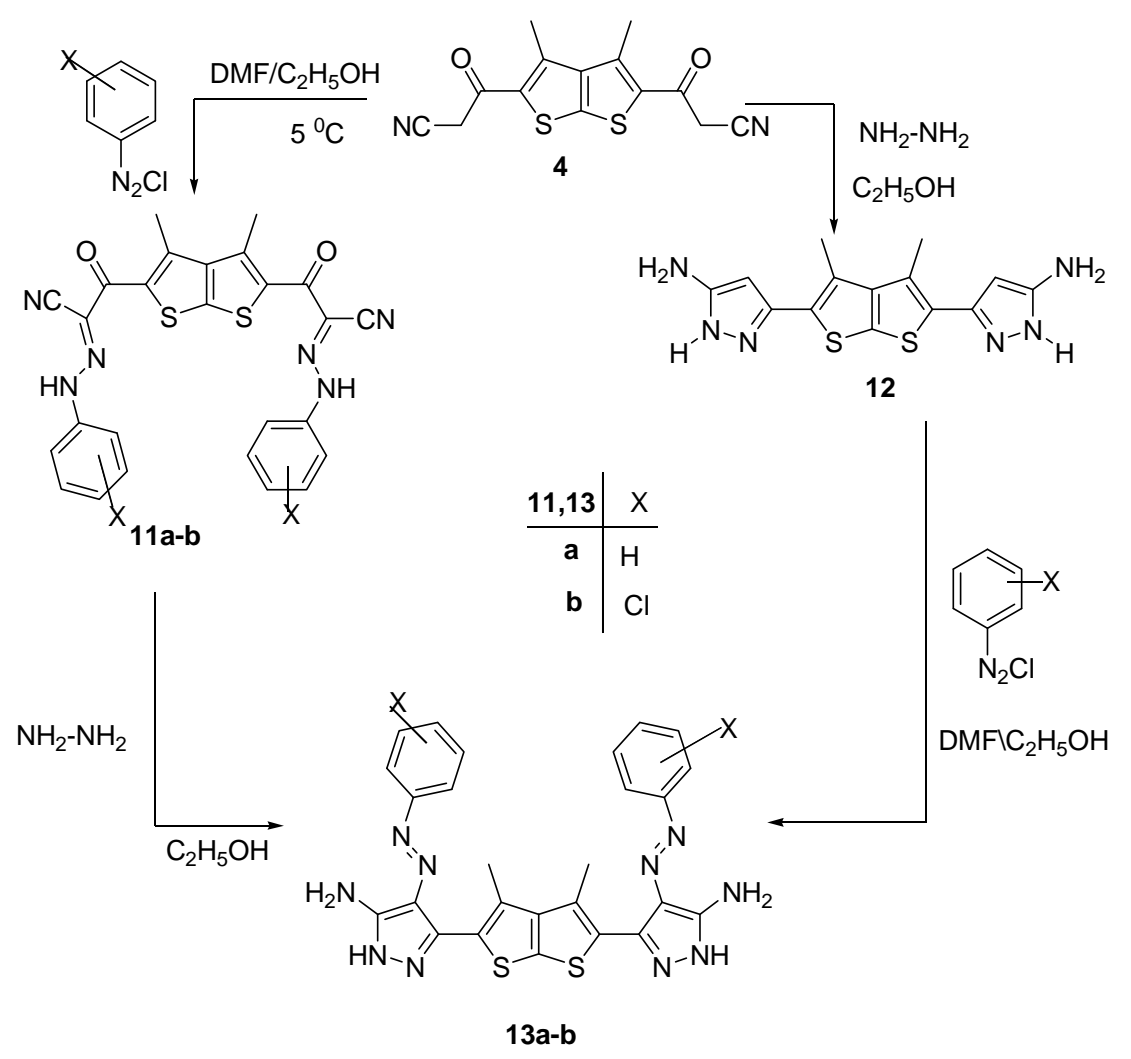

The ${ }^{1} \mathrm{H}-\mathrm{NMR}$ spectrum of 11a, for example, displayed besides an aromatic multiplet at $\delta$ 6.40-7.1 ppm, singlet signals at $\delta 2.22$ and $9.20 \mathrm{ppm}$ corresponding to methyl and hydrazone $\mathrm{NH}$ protons, respectively. Also, the IR spectra showed, in each case, absorption bands at 3200, 2220 and $1716 \mathrm{~cm}^{-1}$ due to $\mathrm{NH}, \mathrm{CN}$ and $\mathrm{CO}$ groups, respectively. The latter hydrazones 11a,b underwent intramolecular cyclization upon treatment with hydrazine hydrate to give products identified as the 3-aminopyrazole derivatives 13a,b. The IR spectrum of 11a,b showed, in each case, the absence of nitrile and carbonyl bands and revealed the appearance of three bands in the $3340-3100 \mathrm{~cm}^{-1}$ region due to $\mathrm{NH}_{2}$ and $\mathrm{NH}$ groups as depicted in Scheme 3.

The ${ }^{13} \mathrm{C}-\mathrm{NMR}$ spectrum of 13a, for example, revealed twelve carbon types. Its ${ }^{1} \mathrm{H}-\mathrm{NMR}$ spectrum displayed singlets at $\delta 4.21$ and $12.7 \mathrm{ppm}$ attributable to the $\mathrm{NH}_{2}$ and $\mathrm{NH}$ protons, respectively. Compounds 13a,b were alternatively obtained by reaction of treatment of bis(aminopyrazole) derivative 12 with diazotized aromatic amines (aniline and $p$-chloroaniline) in cold ethanol/DMF, in 
the presence of sodium acetate trihydrate. Compound 12 was prepared by the reaction of $\mathbf{4}$ with hydrazine hydrate in refluxing ethanol (Scheme 3).

\section{Experimental}

\section{General}

All melting points were measured on a Gallenkamp melting point apparatus. IR spectra were measured as $\mathrm{KBr}$ pellets on a Pye-Unicam SP 3-300 spectrophotometer. The NMR spectra were recorded on a Varian Mercury VX-300 NMR spectrometer. ${ }^{1} \mathrm{H}-\mathrm{NMR}$ (300 MHz) and ${ }^{13} \mathrm{C}-\mathrm{NMR}$ $(75.46 \mathrm{MHz})$ were run in dimethylsulphoxide $\left(\mathrm{DMSO}-\mathrm{d}_{6}\right)$. Chemical shifts were related to that of the solvent. Mass spectra were recorded on a Shimadzu GCMS-QP 1000 EX mass spectrometer at $70 \mathrm{eV}$. Elemental analysis was carried out on an Elementar Vario EL analyzer. Thieno[2,3-b]thiophene derivatives 1,2 were prepared following literature procedures [24,25].

\section{2-Bromo-1-[5-(2-bromoacetyl)-3,4-dimethylthieno[2,3-b]thiophen-2-yl]-ethanone (3)}

A solution of $2(25.2 \mathrm{~g}, 100 \mathrm{mmol})$ in glacial acetic acid $(100 \mathrm{~mL})$ was heated to $80-90{ }^{\circ} \mathrm{C}$ with vigorous stirring. To this hot solution, bromine $(16 \mathrm{~g}, 100 \mathrm{mmol})$ in glacial acetic acid $(20 \mathrm{~mL})$ was added dropwise over a period of $30 \mathrm{~min}$. After complete addition of bromine, the reaction mixture was stirred vigorously at room temperature for further $1 \mathrm{~h}$ till the evolution of hydrogen bromide gas ceased, then it was poured onto crushed ice. The solid that formed was collected, washed with water, dried well and recrystallized from ethanol to give colorless crystals of 3; yield 87\%, mp. $180-182{ }^{\circ} \mathrm{C}$; IR (KBr) $v_{\max } 1690(\mathrm{C}=\mathrm{O}) \mathrm{cm}^{-1} ;{ }^{1} \mathrm{H}-\mathrm{NMR}: \delta 2.23\left(\mathrm{~s}, 6 \mathrm{H}, \mathrm{CH}_{3}\right), 4.82\left(\mathrm{~s}, 4 \mathrm{H}, \mathrm{CH}_{2}\right) ;{ }^{13} \mathrm{C}-\mathrm{NMR}: \delta 9.3$ $\left(\mathrm{CH}_{3}\right.$, aliphatic), $28.50\left(\mathrm{CH}_{2}\right), 132.3,136.1,140.2,148.4$ (ArC's), $186.2(\mathrm{C}=\mathrm{O}) . \mathrm{MS} \mathrm{m} / \mathrm{z}(\%): 407\left(\mathrm{M}^{+}\right.$, 100) , 286 (15), 249 (28), 116 (74); Anal. Calcd. for $\mathrm{C}_{12} \mathrm{H}_{10} \mathrm{Br}_{2} \mathrm{O}_{2} \mathrm{~S}_{2}$ (407.85): C, 35.14; H, 2.46; Br, 38.96; S,15.64. Found: C, 35.03; H, 2.52; Br, 38.85; S, 15.54 .

\section{3-[3,4-Dimethyl-5-(3-nitrilopropanoyl)thieno[2,3-b]thiophen-2-yl]-3-oxopropanenitrile (4)}

Route A: To a mixture of 3,4-dimethylthieno[2,3-b]thiophene-2,5-dicarboxylate (1, $31.24 \mathrm{~g}$, $100 \mathrm{mmol})$ in dry benzene $(200 \mathrm{~mL})$ and dimethylformamide $(10 \mathrm{~mL})$ was added sodium hydride (2.5 g, 60\%). The reaction mixture was refluxed for $4 \mathrm{~h}$, then allowed to cool. The solid that precipitated was collected, washed with ether and dried. The product was dissolved in water and the resulting solution was treated with concentrated hydrochloric acid until it becomes neutral. The precipitated product was collected, washed with water, dried and finally recrystallized from $\mathrm{DMF} / \mathrm{EtOH}$ as colorless crystals; yield 75\%, mp. $254-256{ }^{\circ} \mathrm{C}$; IR (KBr) $v_{\max } 1709$ (C=O), $2214(\mathrm{CN})$ $\mathrm{cm}^{-1}$; ${ }^{1} \mathrm{H}-\mathrm{NMR}: \delta 2.23\left(\mathrm{~s}, 6 \mathrm{H}, \mathrm{CH}_{3}\right), 4.03\left(\mathrm{~s}, 4 \mathrm{H}, \mathrm{CH}_{2}\right) ;{ }^{13} \mathrm{C}-\mathrm{NMR}: \delta 9.3\left(2 \mathrm{CH}_{3}\right.$, aliphatic), 28.50 $\left(2 \mathrm{CH}_{2}\right), 116.3(2 \mathrm{CN}), 131.7,134.5,143.4,160.5$ (ArC's), $193.2(2 \mathrm{C}=\mathrm{O}) ; \mathrm{MS} \mathrm{m} / \mathrm{z}(\%): 303\left(\mathrm{M}^{+}+1\right.$, 100), $302\left(\mathrm{M}^{+}, 85\right), 286$ (17), 249 (19), 160 (34); Anal. for $\mathrm{C}_{14} \mathrm{H}_{10} \mathrm{~N}_{2} \mathrm{O}_{2} \mathrm{~S}_{2}$ (302.37) calcd.: C, 55.61; H, 3.33; N, 9.26; S, 21.21. Found: C, 55.47; H, 3.22 ; N, 9.30 ; S, 21.20.

Route B: Compound 3 (41.01 g, $100 \mathrm{mmol})$ in ethanol $(15 \mathrm{ml})$ was treated with a solution of $\mathrm{KCN}$ ( $6.7 \mathrm{~g}, 100 \mathrm{mmole})$ under reflux for $1 \mathrm{~h}$. The resulting mixture was poured onto cold water. The solid 
precipitate was filtered, dried and recrystallized from DMF/EtOH as colorless crystals, yield $85 \%$. This compound was identical in all respects (mp., mixed mp and spectral data) with compound $\mathbf{3}$ obtained by route A above.

2-(\{5-[2-Cyano-3-(dimethylamino)-2-propenoyl]-3,4-dimethylthieno[2,3-b]thiophen-2-yl $\}$ carbonyl)-3(dimethylamino)-2-propenenitrile (5)

A mixture of bis-3-oxopropanenitrile $4(20 \mathrm{mmol})$ and dimethylformamide-dimethylacetal (DMFDMA, $20 \mathrm{mmol})$ in dry xylene $(20 \mathrm{~mL})$ was refluxed for $8 \mathrm{~h}$, then left to cool to room temperature. The reddish-brown precipitated product was filtered off, washed with light petroleum $\left(40-60{ }^{\circ} \mathrm{C}\right)$, and dried. Recrystallization from $\mathrm{DMF} / \mathrm{EtOH}$ afforded 5. Reddish-brown crystals; Yield 70\%, mp. 290 $292{ }^{\circ} \mathrm{C}$; IR ( KBr) v $v_{\max }, 1713(\mathrm{C}=\mathrm{O}), 2221(\mathrm{CN}), 1554(\mathrm{C}=\mathrm{C}) \mathrm{cm}^{-1}$; ${ }^{1} \mathrm{H}-\mathrm{NMR}: \delta 2.23\left(\mathrm{~s}, 6 \mathrm{H}, \mathrm{CH}_{3}\right), 2.47$ $\left(\mathrm{s}, 12 \mathrm{H}, \mathrm{CH}_{3}\right), 7.51(\mathrm{~s}, 2 \mathrm{H}, \mathrm{CH}) ;{ }^{13} \mathrm{C}-\mathrm{NMR}: \delta 9.2\left(2 \mathrm{CH}_{3}\right.$, aliphatic), $42.7\left(4 \mathrm{CH}_{3}\right), 85.2(2 \mathrm{C}=), 115.9$ (2CN), 131.2, 136.1, 138.1, 147.4 (ArC's), $157.9(2 \mathrm{CH}=) 193.2(2 \mathrm{C}=\mathrm{O}) ; \mathrm{MS} \mathrm{m} / \mathrm{z}(\%): 413\left(\mathrm{M}^{+}+1\right.$, 76), $412\left(\mathrm{M}^{+}, 100\right), 286$ (22), 249 (13); Anal. for $\mathrm{C}_{20} \mathrm{H}_{20} \mathrm{~N}_{4} \mathrm{O}_{2} \mathrm{~S}_{2}$ (412.53) calcd; C, 58.23; H, 4.89; N, 13.58; S, 15.55. Found: C, 58.12; H, 4.64; N, 13.53; S, 15.49.

2-(\{5-[2-Cyano-3-ethoxy-2-propenoyl]-3,4-dimethylthieno[2,3-b] thiophen-2-yl\}carbonyl)-3-ethoxy-2propenenitrile $(\mathbf{6})$

A mixture of bis-3-oxopropanenitrile $4(10 \mathrm{mmol})$, triethylorthoformate $(3 \mathrm{~mL})$ and glacial acetic acid $(3 \mathrm{~mL})$ was refluxed for $3 \mathrm{~h}$. The solid precipitate that formed was filtered off, washed with ethanol, dried and finally recystallized from $\mathrm{DMF} / \mathrm{EtOH}$ afford 5. Yellow crystals; yield 83\%, mp. 282-283 ${ }^{\circ} \mathrm{C}$; IR (KBr) v $v_{\max } 1720(\mathrm{C}=\mathrm{O}), 2205(\mathrm{CN}), 1552(\mathrm{C}=\mathrm{C}) \mathrm{cm}^{-1}$; ${ }^{1} \mathrm{H}-\mathrm{NMR}: \delta 1.22\left(\mathrm{tr}, 6 \mathrm{H}, \mathrm{CH}_{3}\right)$ $2.23\left(\mathrm{~s}, 6 \mathrm{H}, \mathrm{CH}_{3}\right), 4.02$ (q, 4H, $\left.\mathrm{CH}_{2}\right), 7.93(\mathrm{~s}, 2 \mathrm{H}, \mathrm{CH}) ;{ }^{13} \mathrm{C}-\mathrm{NMR}: \delta 9.2\left(2 \mathrm{CH}_{3}\right.$, aliphatic), $15.3\left(2 \mathrm{CH}_{3}\right)$, $64.0\left(2 \mathrm{CH}_{2}\right), 80.1(2 \mathrm{C}=), 115.9(2 \mathrm{CN}), 132.2,136.7,138.9,147.5$, (ArC's), $155.4(2 \mathrm{CH}=), 189.7$ $(2 \mathrm{C}=\mathrm{O}) ; \mathrm{MS} \mathrm{m} / \mathrm{z}(\%): 415.5\left(\mathrm{M}^{+}+1,100\right), 414\left(\mathrm{M}^{+}, 76\right), 286(30), 249(20)$; Anal. for $\mathrm{C}_{20} \mathrm{H}_{18} \mathrm{~N}_{2} \mathrm{O}_{4} \mathrm{~S}_{2}$ (414.5) calcd.; C, 57.95 ; H, 4.38; N, 6.76 ; S, 15.47. Found: C, 57.95; H, 4.38; N, 6.76; S, 15.47.

General procedure for the reaction of bis-thieno[2,3-b]thiophene derivatives 5, 6 with hydrazine derivatives.

Treatment of compounds 5 or 6 ( $1 \mathrm{mmol})$ with hydrazine hydrate and with phenyl hydrazine $(0.1$ $\mathrm{mL})$ in dry ethanol $(15 \mathrm{~mL})$ under reflux for $5 \mathrm{~h}$ afforded the corresponding derivatives 7a,b, respectively. The solid products so formed was filtered off, washed with ethanol, dried and recrystallized from $\mathrm{DMF} / \mathrm{EtOH})$.

(3-Amino-1H-pyrazol-4-yl)\{5-[(3-amino-1H-pyrazol-4-yl)carbonyl]-3,4-dimethylthieno[2,3-b]thiophen-2-yl \}methanone (7a): Colorless crystals; Yield (82\%); mp $>300{ }^{0} \mathrm{C}$; IR (KBr) $v_{\max } 3320,3275$, $3100\left(\mathrm{NH}_{2}+\mathrm{NH}\right), 1735(\mathrm{C}=\mathrm{O}), 1543(\mathrm{CH}=\mathrm{C}) \mathrm{cm}^{-1}$; ${ }^{1} \mathrm{H}-\mathrm{NMR}: \delta 2.22\left(\mathrm{~s}, 6 \mathrm{H}, \mathrm{CH}_{3}\right), 4.92\left(\mathrm{~s}, 4 \mathrm{H}, \mathrm{NH}_{2}\right)$, 7.52 (s, 2H, pyrazole), $9.42(\mathrm{~s}, 2 \mathrm{H}, \mathrm{NH}) ;{ }^{13} \mathrm{C}-\mathrm{NMR}: \delta 9.3\left(2 \mathrm{CH}_{3}\right.$, aliphatic), 94.1, 127.6, 131.2, 136.1, 141.0, 147.5, 161.0 (ArC's),183.7 (C=O); MS m/z (\%): $387\left(\mathrm{M}^{+}+1,100\right) 386\left(\mathrm{M}^{+}, 20\right) 285(30), 251$ (20); Anal. for $\mathrm{C}_{16} \mathrm{H}_{14} \mathrm{~N}_{6} \mathrm{O}_{2} \mathrm{~S}_{2}$ (386.45) calcd.; C, 49.73; H, 3.65; N, 21.75; S, 16.59. Found: C, 49.57; $\mathrm{H}, 3.41 ; \mathrm{N}, 21.55 ; \mathrm{S}, 16.64$. 
(3-Amino-1-phenyl-1H-pyrazol-4-yl)\{5-[(3-amino-1-phenyl-1H-pyrazol-4-yl)carbonyl]-3,4-dimethylthieno[2,3-b] thiophen-2-yl \}methanone (7b): Reddish-brown crystals; Yield (70\%); mp >300 ${ }^{0} \mathrm{C}$; IR $(\mathrm{KBr}) v_{\max }$ 3320, $3275\left(\mathrm{NH}_{2}\right), 1720(\mathrm{C}=\mathrm{O}), 1538(\mathrm{CH}=\mathrm{C}) \mathrm{cm}^{-1} ;{ }^{1} \mathrm{H}-\mathrm{NMR}: \delta 2.22\left(\mathrm{~s}, 6 \mathrm{H}, \mathrm{CH}_{3}\right), 4.92$ (s, $\left.4 \mathrm{H}, \mathrm{NH}_{2}\right), 7.52$ (s, 2H, CH pyrazole), 7.3 (m, 10H ArH's); ${ }^{13} \mathrm{C}-\mathrm{NMR}: \delta 9.3\left(2 \mathrm{CH}_{3}\right.$, aliphatic), 94.1, 120.2, 126.3, 127.6, 129.4, 131.2, 136.1, 139.7, 141.0, 147.5, 161.0 (ArC's), $185.0 \quad(2 \mathrm{C}=\mathrm{O})$; MS m/z (\%): $539\left(\mathrm{M}^{+}+1,55\right), 538\left(\mathrm{M}^{+}, 100\right), 284$ (30), 117 (20); Anal. for $\mathrm{C}_{28} \mathrm{H}_{22} \mathrm{~N}_{6} \mathrm{O}_{2} \mathrm{~S}_{2}(538.64)$ calcd.; C, 62.43; H, 4.12; N, 15.60; S, 11.91. Found: C, 62.39; H, 4.04; N, 15.75; S, 11.78.

6-[5-(7-Cyanopyrazolo[1,5-a]pyrimidin-6-yl)-3,4-dimethylthieno[2,3-b] thiophen-2-yl]-2-phenylpyrazolo[1,5-a]pyrimidine-7-carbonitrile (10)

Method A: To a mixture of the bis-enaminone 5 or bis-ethoxypropenenitrile 6 (10 mmol) and 5-amino-3-phenyl-1H-pyrazole (8a) $(10 \mathrm{mmol})$ in DMF/EtOH $(25 \mathrm{~mL})$ was added few drops of piperidine and the reaction mixture was refluxed for $3 \mathrm{~h}$, then left to cool. The formed solid product was filtered off and recrystallized from EtOH/DMF to afford the pyrazolo-[1,5-a]pyrimidine derivatives 10 in $75 \%$ yield; ${ }^{1} \mathrm{H}-\mathrm{NMR}: \delta 2.22\left(\mathrm{~s}, 6 \mathrm{H}, \mathrm{CH}_{3}\right), 7.36-7.61(\mathrm{~m}, 10 \mathrm{H}, \mathrm{Ph}), 7.79(\mathrm{~s}, 2 \mathrm{H}$, pyrazole), 8.9 (s, $2 \mathrm{H}$, pyrimidine); ${ }^{13} \mathrm{C}-\mathrm{NMR}$ : $\delta 9.3\left(2 \mathrm{CH}_{3}\right.$, aliphatic), $118.2(2 \mathrm{CN}), 92.7,106.5,127.6$, $125.5,127.2,129.1,130.2,130.8,134.5,138.6,148.3,150.3,154.2($ ArC's); MS m/z (\%): 604 $\left(\mathrm{M}^{+}, 100\right), 284$ (29), 253 (25), 115 (61); Anal. for $\mathrm{C}_{34} \mathrm{H}_{20} \mathrm{~N}_{8} \mathrm{~S}_{2}$ (604.71) calcd; C, 67.53; H, 3.33; N, 18.53; S, 10.61. Found: C, 67.68; H, 3.42; N, 18.81; S, 10.77.

Method B: A solution of 3-[3,4-dimethyl-5-(3-nitrilopropanoyl)thieno[2,3-b]thiophen-2-yl]-3oxopropanenitrile $(4,10 \mathrm{mmol})$ and an equivalent molar ratio of 5-N-(N,N-dimethylaminomethylene)amino-3-methyl-1H-pyrazole $(\mathbf{8 b})$ in ethanol $(20 \mathrm{~mL})$, in the presence of piperidine $(0.3 \mathrm{~mL})$, was heated under reflux for $6 \mathrm{~h}$. The solvent was removed by distillation under reduced pressure and the remainder was left to cool. The precipitated solid product was collected by filtration. Recrystallization from $\mathrm{EtOH} / \mathrm{DMF}$ afforded a product identical in all respects (mp, mixed mp, TLC, IR, and mass spectra with 10.

\section{2-Arylhydrazono-3-dimethylthieno[2,3-b]thiophen-3-oxopropanenitriles 11a,b}

To a stirred cold solution of bis-3-oxopropanenitrile 4 (6.04 g, $20 \mathrm{mmol})$ in EtOH/DMF (30 mL) and sodium acetate trihydrate $(2 \mathrm{~g})$, was added the appropriate arene diazonium chloride (20 mmol) portionwise over a period of $30 \mathrm{~min}$ at $0-5^{\circ} \mathrm{C}$. After complete addition, the reaction mixture was stirred for further $3 \mathrm{~h}$ at $0-5^{\circ} \mathrm{C}$. The solid that precipitated was collected, washed with water and dried. Recrystallization from EtOH/DMF afforded the corresponding hydrazones 11a,b.

3-\{5-[5-Amino-4-(2-phenylhydrazino)-1H-pyrazol-3-yl]-3,4-dimethylthieno[2,3-b] thiophen-2-yl\}-4-(2phenylhydrazino)-1H-pyrazo-5-yl amine (11a): Yellow crystals; Yield (80\%); mp. $275^{\circ} \mathrm{C}$; IR (KBr) $v_{\max } 3200(\mathrm{NH}), 2977,2220(\mathrm{CN}), 1716(\mathrm{C}=\mathrm{O}), 1533(\mathrm{CH}=\mathrm{C}) \mathrm{cm}^{-1} ;{ }^{1} \mathrm{H}-\mathrm{NMR}: \delta 2.22\left(\mathrm{~s}, 6 \mathrm{H}, \mathrm{CH}_{3}\right)$, 6.40 -7. 10 (m, 10H, ArH's), 9.20 (s, $2 \mathrm{H}, \mathrm{NH}) ;{ }^{13} \mathrm{C}-\mathrm{NMR}: \delta 9.3\left(2 \mathrm{CH}_{3}\right.$, aliphatic), $115.2(2 \mathrm{CN}), 117.6$, 128.2, 129.8, 132.2, 136.0, 138.2, 142.0, 148.4, (ArC's), $187.2(2 \mathrm{C}=\mathrm{O}) . \mathrm{MS} \mathrm{m} / \mathrm{z}(\%): 511\left(\mathrm{M}^{+}+1\right.$, 
100) $510\left(\mathrm{M}^{+}, 60\right) 286$ (29), 250 (25); Anal. for $\mathrm{C}_{26} \mathrm{H}_{18} \mathrm{~N}_{6} \mathrm{O}_{2} \mathrm{~S}_{2}$ (510.59) calcd.; C, 61.16; H, 3.55; N, 16.46; S, 12.56. Found: C, 60.97; H, 3.64; N, 16.42; S, 12.42.

2-[2-(4-Chlorophenyl)hydrazono]-3-(5-\{2-[2-(4-chlorophenyl)hydrazono]-3-nitrilopropanoyl\}-3,4dimethylthieno[2,3-b]thiophen-2-yl)-3-oxopropanenitrile (11b): Yellow crystals; Yield (85\%); mp. $283^{0} \mathrm{C}$; IR (KBr) v $v_{\max } 3225(\mathrm{NH}), 2220(\mathrm{CN}), 1707(\mathrm{C}=\mathrm{O}), 1521(\mathrm{CH}=\mathrm{C}) \mathrm{cm}^{-1} ;{ }^{1} \mathrm{H}-\mathrm{NMR}: \delta 2.22(\mathrm{~s}$, $\left.6 \mathrm{H}, \mathrm{CH}_{3}\right), 6.24-7.17\left(\mathrm{~m}, 8 \mathrm{H}, \mathrm{ArH}\right.$ 's), $8.21(\mathrm{~s}, 2 \mathrm{H}, \mathrm{NH}) ;{ }^{13} \mathrm{C}-\mathrm{NMR}: \delta 9.2\left(2 \mathrm{CH}_{3}\right.$, aliphatic), 114.9 (2CN), 117.7, 124.3, 129.7, 131.2, 136.1, 138.1, 141.2, 147.5 (ArC's), 184.0 (2C=O); MS m/z (\%): $580\left(\mathrm{M}^{+}+1,100\right), 579\left(\mathrm{M}^{+}, 20\right), 286$ (35), 117 (25); Anal. for $\mathrm{C}_{26} \mathrm{H}_{16} \mathrm{Cl}_{2} \mathrm{~N}_{6} \mathrm{O}_{2} \mathrm{~S}_{2}$ (579.48) calcd.; C, 53.89; H, 2.78; Cl, 12.24; N ,14.50; S, 11.07. Found: C, 53.68; H, 2.69; Cl, 12.52; N, 14.63; S, 10.88.

3-[5-(5-Amino-1H-pyrazol-3-yl)-3,4-dimethylthieno[2,3-b]thiophen-2-yl]-1H-pyrazol-5-yl amine (12)

A mixture of the bis-3-oxopropanenitrile $4(6.04 \mathrm{~g}, 20 \mathrm{mmol})$ and hydrazine hydrate $(1 \mathrm{~mL}, 80 \%)$ in absolute ethanol $(20 \mathrm{ml})$ was refluxed for 2-4 h. The reaction mixture was allowed to cool and then diluted with water. The precipitated solid was collected, washed with water and dried. Recrystallization from EtOH/DMF afforded the pyrazole derivative 12. Colorless crystals; Yield (73\%); mp.285 ${ }^{0} \mathrm{C}$; IR (KBr) $v_{\max } 3320,3250,3120\left(\mathrm{NH}_{2}+\mathrm{NH}\right), 1543(\mathrm{CH}=\mathrm{C}) \mathrm{cm}^{-1} ;{ }^{1} \mathrm{H}-\mathrm{NMR}: \delta 2.21$ (s, 6H, $\left.\mathrm{CH}_{3}\right), 4.02\left(\mathrm{br}, 4 \mathrm{H}, \mathrm{NH}_{2}\right), 6.03\left(\mathrm{~s}, 2 \mathrm{H}\right.$, pyrazole), $12.98(\mathrm{~s}, 2 \mathrm{H}, \mathrm{NH}) ;{ }^{13} \mathrm{C}-\mathrm{NMR}: \delta 9.3\left(2 \mathrm{CH}_{3}\right.$, aliphatic), 88.8, 125.7, 130.2, 133.4, 135.9.0, 138.1.5, 153.0 (ArC's). MS m/z (\%): $331\left(\mathrm{M}^{+}+1,90\right)$, $330\left(\mathrm{M}^{+}, 76\right) 286$ (100), 249 (45); Anal. for $\mathrm{C}_{14} \mathrm{H}_{14} \mathrm{~N}_{6} \mathrm{~S}_{2}$ (330.43) calcd.; C, 50.89; H, 4.27; N, 25.43 ; S, 19.41. Found: C, 50.76; H, 4.26; N, 25.43; S, 19.46.

\section{5-Amino-4-arylazo-3-(thieno[2,3-b]thiophen-2-yl)pyrazoles 13a,b}

Route A: To a solution of the appropriate hydrazone 11a,b $(5 \mathrm{mmol})$ in ethanol $(20 \mathrm{~mL})$ was added hydrazine hydrate $(5 \mathrm{mmol})$. The reaction mixture was refluxed for $1 \mathrm{~h}$, then cooled. The solid formed was collected, washed with ethanol, dried and finally recrystallized from EtOH /DMF to afford the corresponding 4-arylazopyrazole derivatives 13a,b, respec -tively.

Route B: To a stirred cold solution of bis-pyrazole derivative 12 (6.60 g, $20 \mathrm{mmol})$ in EtOH/DMF $(30 \mathrm{~mL})$ and sodium acetate trihydrate $(2 \mathrm{~g})$, was added the appropriate arene diazonium chloride $(20 \mathrm{mmol})$ portionwise over a period of $30 \mathrm{~min}$ at $0-5^{\circ} \mathrm{C}$. After complete addition, the reaction mixture was stirred for further $3 \mathrm{~h}$ at $0-5^{\circ} \mathrm{C}$. The solid that precipitated was collected, washed with water and dried. Recrystallization from EtOH/DMF afforded the corresponding hydrazones 13a,b.

4-(2-Phenyl)diazenyl)-3-(2-(4-(2-phenyl)diazenyl)-5-amino-1H-pyrazol-3-yl)-3,4-dimethylthieno[2,3b] thiophen-2-yl) )-1H-pyrazol-5-amine (13a): Colorless crystals; Yield (45\%); mp. $>300{ }^{0} \mathrm{C}$; IR ( $\left.\mathrm{KBr}\right)$ $v_{\max }$ 3306, 3244, $3100\left(\mathrm{NH}_{2}+\mathrm{NH}\right) \mathrm{cm}^{-1}$; ${ }^{1} \mathrm{H}-\mathrm{NMR}: \delta 2.22\left(\mathrm{~s}, 6 \mathrm{H}, \mathrm{CH}_{3}\right), 4.21$ (br, 4H, $\left.\mathrm{NH}_{2}\right), 6.54-7.17$ (m, 10H, ArH's), 12.7 (s, 2H, NH); ${ }^{13} \mathrm{C}-\mathrm{NMR}: \delta 9.3\left(2 \mathrm{CH}_{3}\right.$, aliphatic), 92.1, 117.2, 121.6, 125.7, 128.5, 128.9, 132.1, 133.8, 138.4, 143.4, 154.0 (ArC's); MS m/z (\%): $539\left(\mathrm{M}^{+}+1,65\right), 538\left(\mathrm{M}^{+}, 100\right)$, 283 (29), 251 (25); Anal. for $\mathrm{C}_{26} \mathrm{H}_{22} \mathrm{~N}_{10} \mathrm{~S}_{2}$ (538.65) calcd; C, 57.97; H, 4.12; N, 26.00; S, 11.91. Found: C, 57.70; H, 4.18; N, 26.15; S, 11.69 . 
4-(2-(4-Chlorophenyl)diazenyl)-3-(2-(4-(2-(4-chlorophenyl)diazenyl)-5-amino-1H-pyrazol-3-yl)-3,4dimethylthieno[2,3-b] thiophen-2-yl) )-1H-pyrazol-5-amine (13b): Colorless crystals; Yield (60\%); mp. $>300{ }^{0} \mathrm{C}$; IR $(\mathrm{KBr}) v_{\max } 3333,3221,3150\left(\mathrm{NH}_{2}+\mathrm{NH}\right) \mathrm{cm}^{-1} ;{ }^{1} \mathrm{H}-\mathrm{NMR}: \delta 2.22\left(\mathrm{~s}, 6 \mathrm{H}, \mathrm{CH}_{3}\right), 4.00$ (br, 4H, $\mathrm{NH}_{2}$ ), 7. 20-7.32 (m, 8H, ArH's), 13.7 (s, 2H, NH); ${ }^{13} \mathrm{C}-\mathrm{NMR}: \delta 9.3\left(2 \mathrm{CH}_{3}\right.$, aliphatic), 92.1, 125.8, 126.6, 125.7, 128.5, 130.2, 130.3, 133.7, 134.3, 138.4, 154.0, (ArC's); MS m/z (\%): 608 (M+1, 100), 607 ( $\left.\mathrm{M}^{+}, 54\right), 286$ (29), 115 (25); Anal. for $\mathrm{C}_{26} \mathrm{H}_{20} \mathrm{Cl}_{2} \mathrm{~N}_{10} \mathrm{~S}_{2}$ (607.54) calcd.; C, 51.40; H, 3.32; Cl, 11.67; N, 23.05; S, 10.56. Found: C, 51.78; H, 3.26; Cl, 11.84; N, 23.17; S, 10.55.

\section{Conclusions}

Synthesis and identification of some bis-heterocycles 7a,b, 10, 12 and 13a,b containing thieno[2,3b]thiophene as a base unit via the versatile, hitherto unreported 3-[3,4-dimethyl-5-(3nitrilopropanoyl)thieno[2,3-b]thiophen-2-yl]-3-oxopropanenitrile(4) was reported.

\section{Acknowledgements}

The author is grateful to King Saud University [supported by College of Science-Research Center project No. (Chem./2008/66)].

\section{References}

1. Eicher, T.; Hauptmann, S.; Speicher, A. Five-Membered Heterocycle. In The Chemistry of Heterocycles; Wiley-VCH: New York, NY, USA, 2003.

2. Gronowitz, S. The Chemistry of Heterocyclic Compounds: Thiophene and its Derivatives; Gronowitz, S., Ed.; Wiley: New York, NY, USA, 1991; Vol. 44, Part 3, Chapter 2.

3. King, W.J.; Nord, F.F. Studies In The Thiophene Series. V. Wolff-Kishner Reductions. J. Org. Chem. 1949, 14, 638-642.

4. Wu, C.; Decker, E.R.; Blok, N.; Bui, H.; You, T.J.; Wang, J.; Bourgoyne, A.R.; Knowles, V.; Berens, K.L.; Holland, G.W.; Brock, T.A.; Dixon, R.A.F. Discovery, Modeling, and Human Pharmacokinetics of $N$-(2-Acetyl-4,6-dimethylphenyl)-3-(3,4-dimethylisoxazol-5-ylsulfamoyl)thiophene-2-carboxamide (TBC3711), a Second Generation, ET $_{\mathrm{A}}$ Selective, and Orally Bioavailable Endothelin Antagonist. J. Med. Chem. 2004, 47, 1969-1986.

5. Dubus, S.; Levesque, I.; Brunette, M.; Corbeil, G.; Boissinot, M.; Boivin, G.; Bergeron, M.G.; Boudreau, D.; Leclerc, M. Fluorescent Polymeric Transducer for the Rapid, Simple, and Specific Detection of Nucleic Acids at the Zeptomole Level. J. Am. Chem. Soc. 2004, 126, 4240-4244.

6. Rost, C.; Karg, S.; Riess,W.; Loi, M.A.; Murgia, M.; Muccini, M. Ambipolar light-emitting organic field-effect transistor. Appl. Phys. Lett. 2004, 85, 1613-1615.

7. Vriezema, D.M.; Hoogboom, J.; Velonia, K.; Takazawa, K.; Christianen, P.C.M.; Maan, J.C.; Rowan, A.E.; Nolte, R.J. Vesicles and Polymerized Vesicles from Thiophene-Containing RodCoil Block Copolymers. Angew. Chem. Int. Ed. 2003, 42, 772-776.

8. Pullen, A.E.; Bushel, M.G.; Swager, T.M. Charge-Specific Interactions in Segmented Conducting Polymers: An Approach to Selective Ionoresistive Responses. Angew. Chem. Int. Ed. 2004, 43, 3700-3703. 
9. Heeney, M.; Bailey, C.; Genevicius, K.; Shkunov, M.; Sparrowe, D.; Tierney, S.; Mculloch, I. Stable Polythiophene Semiconductors Incorporating Thieno[2,3-b]thiophene. J. Am. Chem. Soc. 2005, 127, 1078-1079.

10. Mashraqui, S.H; Sangvikar, Y.S; Meetsma, A. Synthesis and structures of thieno[2,3-b]thiophene incorporated [3.3]dithiacyclophanes. Enhanced first hyperpolarizability in an unsymmetrically polarized cyclophane. Tetrahedron Lett. 2006, 47, 5599-5602.

11. Mashraqui, S.H.; Sangvikar, Y.; Ashraf, M.; Kumar, S.; Daub, E. Dipyridyl/pyridinium thieno[2,3-b]thiophenes as new atropisomeric systems. Synthesis, conformat-ional analysis and energy minimization. Tetrahedron 2005, 61, 3507-3513.

12. Leriche, P.R.J.; Turbiez, M.M.; Monroche, V.; Allain, M.; Sauvage, F.X.; Roncali, J.; Frere, P.; Skabara, P.J. Linearly extended tetrathiafulvalene analogues with fused thiophene units as $\pi$ conjugated spacers. J. Mater. Chem. 2003, 13, 1324-1327.

13. Lee, B.; Seshadri, V.; Palko, H.; Sotzing, G.A. Ring-Sulfonated Poly(thienothiophene. J. Adv. Mater. 2005, 17, 1792-1795.

14. Lim, E.; Jung, B.J.; Lee, J.; Shim, H.K.; Lee, J.I.; Yang, Y.S.; Do, L.M. Thin-Film Morphologies and Solution-Processable Field-Effect Transistor Behavior of a Fluorene-Thieno[3,2b]thiophene-Based Conjugated Copolymer. Macromolecules 2005, 38, 4531-4535.

15. Kim, H.S.; Kim, Y.H.; Kim, T.H.; Noh, Y.Y.; Pyo, S.; Yi, M.H.; Kim, D.Y.; Kwon, S.K. Synthesis and Studies on 2-Hexylthieno[3,2-b]thiophene End-Capped Oligomers for OTFTs. Chem. Mater. 2007, 19, 3561-3567.

16. Jarak, I.; Kralj, M.; Piantanida, I.; Suman, L.; Zinic, M.; Pavelic, K.; Karminski-Zamola, G. Novel cyano- and amidino-substituted derivatives of thieno[2,3-b]- and thien- o[3,2-b]thiophene2-carboxanilides and thieno[30,20:4,5]thieno- and thieno [20,30:4,5] thieno[2,3-c]quinolones: Synthesis, photochemical synthesis, DNA binding, and antitumor evaluation. Bioorg. Med. Chem. 2006, 14, 2859-2868.

17. Peters, D.; Hornfeldt, A.B.; Gronowitz, S. Synthesis of various 5-substituted uracils. J. Heterocycl. Chem. 1990, 27, 2165-2173.

18. Kukolja, S.; Draheim, S.E.; Graves, B.J.; Hunden, D.C.; Pfeil, J.L.; Cooper, R.D.G.; Ott, J.L.; Couter, F.T. Orally absorbable cephalosporin antibiotics. 2. Structure-activity studies of bicyclic glycine derivatives of 7-aminodeacetoxycephalosporanic acid. J. Med. Chem. 1985, 28, 18961903.

19. Prugh, J.D.; Hartman, G.D.; Mallorga, P.J.; McKeever, B.M.; Michelson, S.R.; Murcko, M.A.; Schwam, H.; Smith, R.L.; Sondey, J.M.; Springer, J.P.; Surgrue, M.F. New isomeric classes of topically active ocular hypotensive carbonic anhydrase inhibitors: 5-substituted thieno[2,3b]thiophene-2-sulfonamides and 5-substituted thieno[3,2-b]thiophene-2-sulfonamides. J. Med. Chem. 1991, 34, 1805-1818.

20. Egbertson, M.S.; Cook, J.J.; Bednar, B.; Prugh, J.D.; Bednar, R.A.; Gaul, S.L.; Gould, R.J.; Hartman, G.D.; Homnick, C.F.; Holahan, M.A.; Libby, L.A.; Lynch, J.J., Jr.; Lynch, R.J.; Sitko, G.R.; Stranieri, M.T.; Vassallo, L.M. Non-Peptide GPIIb/IIIa Inhibitors. 20. Centrally Constrained Thienothiophene $\alpha$-Sulfonamides Are Potent, Long Acting in Vivo Inhibitors of Platelet Aggregation. J. Med. Chem. 1999, 42, 2409-2421. 
21. Schuett, W.; Raoort, H. Saxitoxin, the Paralytic Shellfish Poison. Degradation to a Pyrrolopyrimidine. J. Am. Chem. Soc. 1962, 84, 2266-2267.

22. Alexander, S.K.; Smith, L. Novel one pot synthesis of polysubstituted pyrazolo[1,5-a] and imidazo[1,2-a]pyrimidines. Tetrahedron Lett. 2006, 47, 2611-2614.

23. Shaaban, M.R.; Saleh, T.S.; Mayhoub, A.; Farag, A.M. Synthesis and analgesic/ antiinflammatory evaluation of fused heterocyclic ring systems incorporating phenylsulfonyl moiety. Bioorg. Med. Chem. 2008, 16, 6344-6352.

24. Comel, A.; Kirsch, G. Efficient one pot preparation of variously substituted thieno [2,3-b] thiophene. J. Heterocycl. Chem. 2001, 38, 1167-1171.

25. El-Saghier, M.A. Simple One-Pot Synthesis of Thieno[2,3-b]thiophene Derivatives under SolidLiquid PTC Conditions. Useful Starting Material for the Synthesis of Biological Active Compounds. Bull. Chem. Soc. Jpn. 1993, 66, 2011-2015.

Sample Availability: Samples of the compounds are available from the author.

(C) 2009 by the authors; licensee Molecular Diversity Preservation International, Basel, Switzerland. This article is an open-access article distributed under the terms and conditions of the Creative Commons Attribution license (http://creativecommons.org/licenses/by/3.0/). 